\title{
Laparoscopic selective esophagogastric devascularization and splenectomy for patients with cirrhotic portal hypertension
}

\author{
Jie Lin, Qingbo Liu, Zhiqiang Liang, Wei He, Jianping Chen, Jing Ma, Chichang Gu, Weidong Wang \\ Second Department of General Surgery, Shunde Hospital, Southern Medical University (The First People's Hospital of Shunde Foshan), \\ Foshan, China
}

Videosurgery Miniinv 2019; 14 (2): 187-194

DOI: https://doi.org/10.5114/wiitm.2018.80297

\begin{abstract}
Introduction: The role of laparoscopic selective esophagogastric devascularization and splenectomy (LSEGDS) in the treatment of esophagogastric variceal bleeding and hypersplenism in patients with cirrhotic portal hypertension has not been well studied.

Aim: To investigate the safety and efficacy of LSEGDS for esophagogastric variceal bleeding and hypersplenism in patients with cirrhotic portal hypertension.

Material and methods: From May 2011 to December 2014, 74 patients with portal hypertension resulting from liver cirrhosis underwent surgery for gastroesophageal variceal bleeding and hypersplenism. Forty-one of these patients underwent laparoscopic esophagogastric devascularization and splenectomy (LEGDS), and the others underwent LSEGDS. A retrospective comparative analysis of clinical data was conducted between the two groups, including clinical characteristics, laboratory data, operative morbidity and mortality, and outcomes of follow-up.

Results: The operation was completed successfully in all the patients, except that conversion was required in one patient in the LEGDS group. The operating time was similar in both groups $(p=0.579)$. The intraoperative blood loss was lower in the LSEGDS group ( $p=0.011$ ). Postoperative complications showed no significant difference between the two groups regarding mortality rate, pleural effusion, pancreatic injury, pulmonary infection, liver dysfunction, or postoperative abdominal bleeding. Postoperative platelet counts increased significantly more in the LEGDS group than in the LSEGDS group ( $p=0.004)$. There were no significant differences in the long-term follow-up data, such as incidence of rebleeding, portal vein thrombosis, hepatic encephalopathy and survival ( $p>0.05)$.

Conclusions: The LSEGDS is a safe and effective procedure for management of cirrhotic portal hypertension, especially in patients with visible paraesophageal veins.
\end{abstract}

Key words: liver cirrhosis, portal hypertension, splenectomy, laparoscopic treatment, selective periesophagogastric devascularization.

\section{Introduction}

It is well known that portal hypertension resulting from liver cirrhosis generally leads to two severe complications: bleeding from esophagogastric varices, and thrombocytopenia due to hypersplenism. Bleeding from esophagogastric varices is a major cause of death in patients with cirrhosis. The variceal bleeding mortality is reported to be about $20 \%$ in 6 weeks despite improvement in nonsurgical therapy over the last decade [1]. Endoscopic therapy can effectively control most cases of acute variceal bleeding, but its long-term results are far from satisfactory. The risk of recurrent bleeding is as high as

\section{Address for correspondence}

Weidong Wang MD, Second Department of General Surgery, Shunde Hospital, Southern Medical University (The First People's Hospital of Shunde Foshan), Lunjiao St, Shunde District, 528300 Foshan, China, phone: +86 757 22318705, e-mail: weidongwang1968@163.com 
$60 \%$ [2]. In addition, severe thrombocytopenia due to hypersplenism significantly increases the risk of spontaneous bleeding. As a result, surgical intervention still plays an important role in patients with cirrhotic portal hypertension, and remains the most reliable treatment for esophagogastric varices bleeding and hypersplenism.

Two surgical procedures have been widely applied: esophagogastric devascularization and splenectomy (EGDS) and distal splenorenal shunt (DSRS). In recent years, with significant advances in laparoscopic instruments and techniques, laparoscopic esophagogastric devascularization and splenectomy (LEGDS) has been reported as a safe and effective procedure for portal hypertension, with the advantages of minimally invasive surgery [3]. In order to improve the efficacy of the devascularization procedure, a new surgical strategy is required. Selective esophagogastric devascularization and splenectomy (SEGDS) is being considered as a new choice [4]. However, few studies on the laparoscopic procedure have been published.

\section{Aim}

The aim of the present study was to investigate the safety, effectiveness and outcomes of laparoscopic selective esophagogastric devascularization and splenectomy (LSEGDS) in patients with portal hypertension secondary to liver cirrhosis.

\section{Material and methods}

\section{Clinical characteristics of patients}

From January 2011 to December 2014, a series of 74 patients (Table I) with portal hypertension of cirrhotic origin underwent surgery for esophagogastric varices bleeding and hypersplenism in the department. Forty-one patients were treated with LEGDS, and the others underwent LSEGDS. The surgical indications were as follows: (1) portal hypertension of cirrhotic origin, with a history of esophagogastric varices bleeding; and (2) hypersplenism and splenomegaly (platelet count $\leq 50 \times 10^{9} / /$ or white blood cell count $\leq 3 \times 10^{9} / \mathrm{l}$ ); and (3) Child-Pugh class A and $B$, or class $C$ with hematemesis that cannot be controlled effectively by endoscopic therapy. All of the operations were performed by the same surgeon.

All patients underwent detailed clinical evaluation and biochemical investigation. Preoperative computed tomography $(\mathrm{CT})$ provides information of the size of the liver and spleen, and details of splenic vascular anatomy, and confirms the presence or absence of ascites. Computed tomography image reconstruction was done to evaluate the portal vein system and collateral circulation. Prior to surgery, supportive measures to improve coagulation, blood loss, liver function, and nutrition were implemented in all patients. Esophagogastroscopy was performed to document the presence of esophageal and gastric varices, as well as portal hypertension gastropathy. All patients were grouped according to the ChildPugh classification and the varices were graded according to Conn's classification. $\beta$-Blockers were prophylactically administered to all patients to decrease portal pressure and the risk of rebleeding. Preoperative clinical data, intraoperative and postoperative details, and outcomes of follow-up were retrospectively analyzed. All study participants, or their legal guardian, provided informed written consent prior to study enrollment. This study was reviewed and approved by the Ethics Committee of our hospital.

\section{Surgical technique}

After general anesthesia, the patient was placed in the right semilateral recumbent position with the left side elevated $30^{\circ}$ and the reverse Trendelenburg position at $30^{\circ}$. Generally, four ports were used as follows. Port A: a $10-\mathrm{mm}$ trocar was placed in the umbilical region for the $30^{\circ}$ laparoscope. A pneumoperitoneum was set up using carbon dioxide at a pressure of $12-14 \mathrm{~mm} \mathrm{Hg}$ through port A. Port B: a $10-12-\mathrm{mm}$ trocar was placed at the mid-point of the line between the appendix ensiformis and the umbilicus. At/below the level of the umbilicus, port $C$ with a 5-mm trocar and port D with a 10-12-mm trocar were placed in the left midclavicular line and in the left anterior axillary line, respectively, depending on the size of the spleen. Through ports B and D, the surgeon could perform the major operation, while port $C$ was used for auxiliary operations.

Splenectomy was performed using a Ligasure Vessel Sealing System and Harmonic Scalpel (Ethicon Endo-Surgery). The left side of the gastrocolic and splenogastric ligaments, including the short gastric vessel, were divided to open the lesser omental bursa. The splenic artery was isolated by electrocautery or Harmonic Scalpel, and ligated with a Hem-o-lok clamp. At this point, the volume of the enlarged spleen decreased with blood drainage. There were two ways to 
Table I. Preoperative clinical data of patients

\begin{tabular}{|c|c|c|c|c|}
\hline Variables & Group $1(n=41)$ & Group $2(n=33)$ & $\mathrm{W} / \chi^{2}$ & $P$-value \\
\hline Age, median (min., max.) [years] & $52(30,68)$ & $49(25,77)$ & 1203.5 & 0.711 \\
\hline Gender: & & & 1.094 & 0.296 \\
\hline Male & 36 & 26 & & \\
\hline Female & 5 & 7 & & \\
\hline HBV infection: & & & 1.22 & 0.269 \\
\hline Yes & 32 & 29 & & \\
\hline No & 9 & 4 & & \\
\hline Child-Pugh class: & & & 0.403 & 0.525 \\
\hline A & 27 & 24 & & \\
\hline $\mathrm{B}$ & 13 & 9 & & \\
\hline C & 1 & 0 & & \\
\hline History of varices bleeding episodes: & & & 0.001 & $1.000^{\mathrm{a}}$ \\
\hline More than once & 35 & 28 & & \\
\hline Once & 6 & 5 & & \\
\hline History of endoscopy therapy: & & & 0.016 & 0.898 \\
\hline Yes & 23 & 19 & & \\
\hline No & 18 & 14 & & \\
\hline Emergency surgery: & & & & $1.000^{b}$ \\
\hline Yes & 1 & 0 & & \\
\hline No & 40 & 33 & & \\
\hline Grade of varices: & & & 1.100 & 0.294 \\
\hline Grade IV & 35 & 25 & & \\
\hline Grade III & 6 & 8 & & \\
\hline Main site of severe varices: & & & 0.865 & 0.649 \\
\hline Esophageal & 24 & 16 & & \\
\hline Fundus & 8 & 7 & & \\
\hline Both & 9 & 10 & & \\
\hline Diagnosis: & & & 0.292 & 0.589 \\
\hline Posthepatitis cirrhosis & 33 & 29 & & \\
\hline Alcoholic cirrhosis & 8 & 4 & & \\
\hline
\end{tabular}

${ }^{a} 1$ cell had expected count less than 5 , continuity correction was applied. ${ }^{b} 2$ cells had expected count less than 1 , Fisher's exact test was applied.

deal with the splenic hilum. One way was that the splenic hilum was transected with the linear laparoscopic vascular stapler (Endo-GIA) after the spleen was freed from all the attachments and ligaments. The other way was dividing all the secondary vessels of the splenic pedicle one by one using Hem-o-lok clamps and Ligasure Vessel Sealing System or Harmonic Scalpel until the splenic pedicle was dissected completely. The spleen was then pushed to the right with the aid of a retractor. All of the attachments and 
ligaments were dissected, including splenocolic, splenorenal, splenogastric and lienophrenic ligaments. The spleen was placed into a retrieval bag, morcellated with forceps, and pulled out through port B.

After splenectomy, periesophagogastric devascularization began with dissection of the vena gastrica posterior. Subsequently, the gastrohepatic ligament was opened, permitting identification and isolation of the left gastric vein. The left gastric vein was doubly ligated with a Hem-o-lok. After the left gastric vein was disconnected, the varices at the lesser curvature of the stomach were dissected toward the distal esophagus in the gastric serosa through the posterior gastric approach. Devascularization of the greater curvature varices was performed by the same method. The varices were devascularized with Hem-o-lok clamps and Harmonic Scalpel or LigaSure Vessel Sealing System, including gastric and esophageal perforating branches and superior position perforating branches. In selective devascularization, the gastric branch of the left gastric vein was ligated and disconnected instead of the stem of the left gastric vein, and perforating branches were disconnected through anterior and posterior gastric approaches. Meanwhile, the esophageal branch of the left gastric vein (paraesophageal vein) was preserved (Photo 1). Therefore, both the gastric coronary vein and paraesophageal collateral veins toward the azygous shunt were reserved [4]. Laparoscopic devascularization was accomplished, with complete freeing of at least $8 \mathrm{~cm}$ of the distal esophagus.

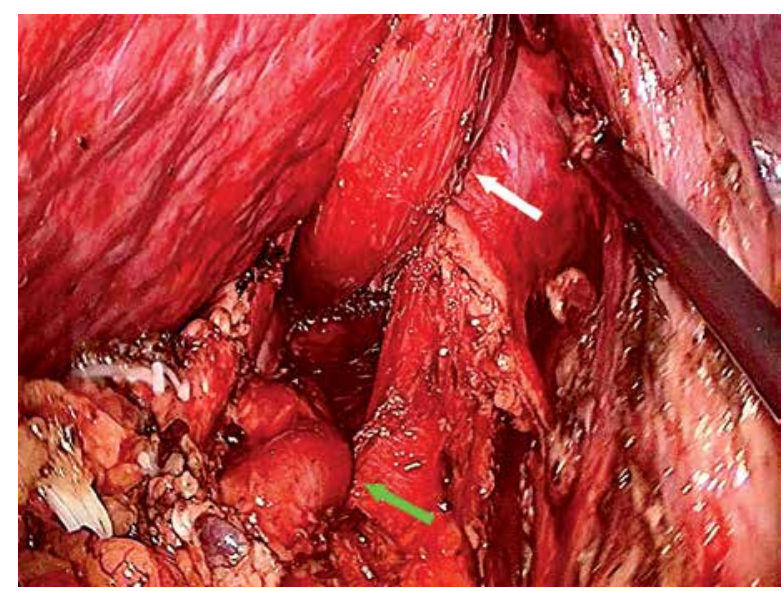

Photo 1. Preserved paraesophageal vein during operation. Green arrowhead points to paraesophageal vein and white arrowhead points to esophagus

\section{Statistical analysis}

Continuous variables were expressed as mean \pm standard deviation and compared using Student's $t$ test. The $\chi^{2}$ test was used to compare categorical variables. The survival rates were calculated using Kaplan-Meier plots and compared using the log-rank test between different groups. All statistical tests were two-sided, and $p<0.05$ was considered statistically significant. All analyses were conducted using SPSS version 13.0 for Windows.

\section{Results}

\section{Comparison of clinical characteristics in LEGDS and LSEGDS groups}

The characteristics of the patients in the LEGDS group (group 1) and LSEGDS group (group 2) and comparisons between them are shown in Table I. No significant differences were found in preoperative data between patients in the two groups.

\section{Operative details}

The laparoscopic procedure was successfully performed in all patients, except that conversion was required in 1 patient in group 1 due to uncontrolled intraoperative bleeding from the splenic fossa after splenectomy. The operation time was similar for the two groups (265 min vs. $275 \mathrm{~min}$ ). However, blood loss in group 2 was much lower than that in group 1 $(100 \mathrm{ml}$ vs. $200 \mathrm{ml}, p=0.011)$. In addition, no significant differences were found in terms of conversion rate, blood transfusion, spleen weight, and additional operation (Table II).

\section{Postoperative complications}

The operative mortality was defined as death within 30 days after the operation and was $2.1 \%(1 / 41)$ in group 1 compared with $3.0 \%(1 / 33)$ in group 2 , which had no significant difference $(p>0.05)$. In addition, the overall complication rate showed no significant difference between the two groups $(p=0.354)$. Twenty patients who underwent LSEGDS experienced one or more early postoperative complications: 4 with pleural effusion, 2 with pancreatic injury (marked increase in serum amylase), 5 with pulmonary infection, 2 with liver dysfunction, and 2 with postoperative bleeding. The patient with pancreatic injury did not have pancreatic leakage. Of the patients who underwent LEGDS, 3 had pleural effusion, 2 had 
Table II. Intraoperative details in the two groups

\begin{tabular}{|c|c|c|c|c|}
\hline Variables & Group $1(n=41)$ & Group $2(n=33)$ & $W / \chi^{2}$ & $P$-value \\
\hline Operation time (min., max.) [min] & $265(120,430)$ & $275(105,385)$ & 1486.5 & 0.579 \\
\hline Estimated blood loss (min., max.) [ml] & $200(50,1200)$ & $100(50,800)$ & 1005.0 & 0.011 \\
\hline Spleen weight (min., max.) [g] & $685.0(177.0,2353.0)$ & $875.0(259.0,1999.0)$ & 1390.0 & 0.109 \\
\hline Transfusion (RBC): & & & 0.622 & 0.430 \\
\hline Yes & 12 & 7 & & \\
\hline No & 29 & 26 & & \\
\hline Conversion: & & & & $0.554^{\mathrm{a}}$ \\
\hline Yes & 1 & 0 & & \\
\hline No & 40 & 33 & & \\
\hline Additional operation (LC): & & & 0.217 & 0.641 \\
\hline Yes & 14 & 13 & & \\
\hline No & 27 & 20 & & \\
\hline
\end{tabular}

a cell had expected count less than 1, Fisher's exact test was applied.

pulmonary infection, and 2 had postoperative bleeding. The patients who had these postoperative complications, except postoperative abdominal bleeding, were all cured by conservative treatment. The patient in group 1 who had postoperative abdominal bleeding that induced disseminated intravascular coagulation died on postoperative day (POD) 11. In group 2, 1 patient who had postoperative abdominal bleeding needed emergency laparoscopic surgery and blood transfusion, and was cured. However, another patient needed emergency traditional laparotomy for bleeding, and died of multiple organ dysfunction syndrome on POD 20. There were no episodes of rebleeding during the hospital stay.

\section{Clinical and laboratory data}

At the first week after the operation, compared with group 2, postoperative platelet count in group 1 increased significantly postoperatively $\left(282 \times 10^{9} / \mathrm{l}\right.$ vs. $\left.210 \times 10^{9} / \mathrm{l}, p=0.004\right)$. Liver function showed no significant changes between the two groups before and after the operation, including total bilirubin level $(20.3 \mu \mathrm{mol} / \mathrm{l}$ vs. $19.8 \mu \mathrm{mol} / \mathrm{l}, p=0.798)$ and prothrombin time (14.3 s vs. $14.7 \mathrm{~s}, p=0.424)$.

\section{Follow-up outcomes}

All patients were available for follow-up, except the patients who died in the intraoperative period. The median follow-up was 28.0 months (range: 0-52.0 months). The severity of varices was attenuated in all the patients compared with that preoperatively. There were no significant differences between the two groups in rebleeding, portal vein thrombosis (PVT), hepatic encephalopathy, hepatocellular carcinoma, and gastric retention. Six patients experienced rebleeding in group 1: 4 with esophagogastric varices, 1 with portal hypertension gastropathy, and 1 with gastric ulcer. Four patients experienced rebleeding in group 2: 2 with esophagogastric varices, 1 with portal hypertension gastropathy, and 1 with gastric ulcer. Most symptoms of rebleeding were mild and were managed conservatively. Most patients with PVT had no symptoms, and their condition was not life-threatening.

\section{Survival}

The median follow-up time was 30.0 (0-52.0) months in group 1 and 25.0 (0-51.0) months in group 2. The 1- and 3-year survival rates were $97.6 \%$ and $88.5 \%$ in group 1 and $97.0 \%$ and $97.0 \%$ in group 2, respectively. The survival curves are shown in Figure 1 and the log-rank test revealed no significant difference between the two groups ( $p=0.537)$.

\section{Discussion}

The LEGDS for portal hypertension was first described in 1998 [5]. Due to technical difficulties, 


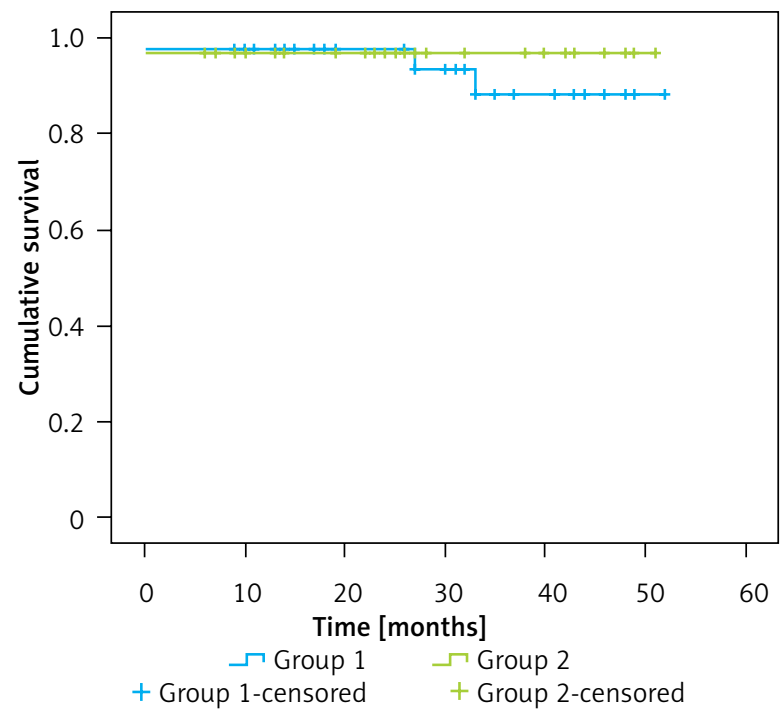

Figure 1. Survival curves of the two groups of patients

LEGDS has been considered a high-risk operation, and a hand-assisted laparoscopic procedure was recommended [6]. Recently, with significant advances in laparoscopic instruments and techniques, LEGDS has been adopted as a safe and effective procedure for treatment of patients with portal hypertension, with favorable results [7-9]. Our study not only confirmed the safety of LEGDS for cirrhotic portal hy-

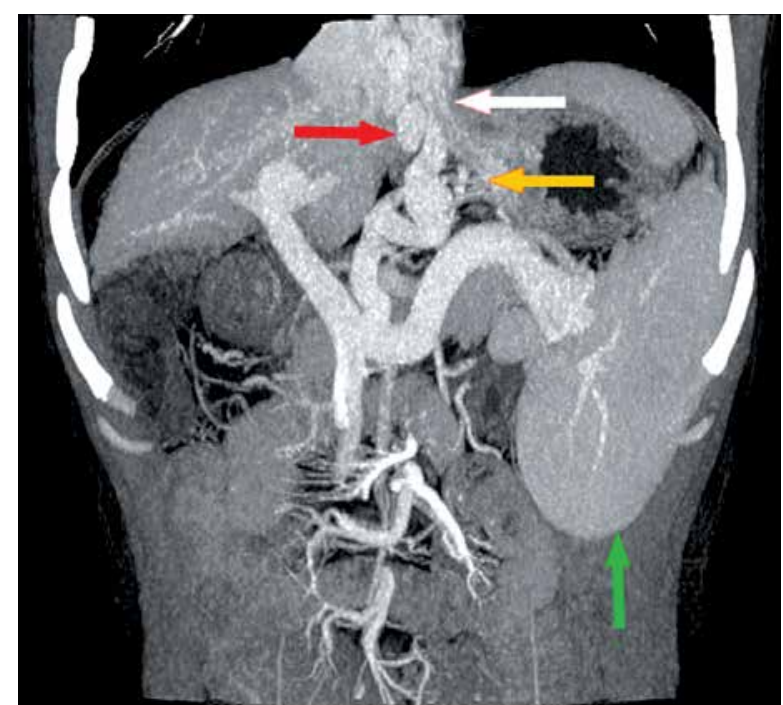

Photo 2. Preoperative CT image reconstruction of the portal vein system. White arrowhead points to esophagus; red arrowhead points to paraesophageal vein; yellow arrowhead points to perforating branch; and green arrowhead points to spleen pertension, but also showed the efficacy of LSEGDS, which has rarely been reported.

In this study, all the patients had endoscopic checkup before surgery, while for a lot of patients we could not find the active bleeding during the procedure, which is why only $56.1 \%(23 / 41)$ of patients in the non-selective surgery group and $57.6 \%$ (19/33) of patients in the selective surgery group had endoscopic treatment. Most of our patients were infected with hepatitis B virus (HBV), as shown in Table I (78\%, and $87.9 \%$ respectively). The pathophysiology of these portal hypertension patients is different from that of patients with etiologies such as ethanol abuse or parasite infection. Even though endoscopic therapy can effectively control most cases of acute variceal bleeding, the long-term recurrent bleeding rate is as high as $60 \%$ [2]. In addition, most of the patients were compromised with severe thrombocytopenia, which increased the risk of re-bleeding. Since splenectomy offers the chance of alleviating severe thrombocytopenia and devascularization removes the variceal plexus, we chose both procedures simultaneously to remove both risks.

Laparoscopic splenectomy (LS) is a promising procedure for treatment of hypersplenism and splenomegaly secondary to portal hypertension, and plays a key role in laparoscopic devascularization [10-13]. It not only provides enough working space for the following devascularization procedure, but also removes the diseased spleen and thus improves the thrombocytopenia.

To improve devascularization, we performed LEGDS on patients with portal hypertension and esophagogastric varices bleeding. Selective devascularization, which retains a spontaneous shunt of the portal vein and the blood flow direction from the gastric coronary vein to the semi-azygos vein, is considered an ideal surgical approach for treatment of portal hypertension $[4,14]$. It not only decreases the portal vein pressure, but also preserves sufficient portal vein perfusion to protect liver function. In theory, that is considered to have the advantages of both the shunt and devascularization procedures.

In the present study, we proved that LSEGDS was a safe and technically feasible procedure. There are several essential points we want to share while performing this procedure. First, the key is to identify and preserve the paraesophageal vein. Preoperative CT image reconstruction of the portal vein system can reveal the tortuous paraesophageal vein clearly (Photo 2). Laparoscopy offers the surgeon a good vi- 
sual field and operating space. We discovered that the paraesophageal vein is usually thick and tortuous. It is not fixed at a $0.5 \mathrm{~cm}$ distance from the esophagus, which is not in conformity with what was described previously [15]. In addition, it adjoins the esophageal wall after entering the esophageal hiatus. Furthermore, esophageal perforating branch vessels do not enter the esophageal wall vertically after running from the paraesophageal vein. They follow along the esophagus for a certain distance and then enter the esophagus. This makes it possible to separate the paraesophageal vein from the esophagus while pulling the esophagus and its surrounding tissues towards the other side. Second, we found that gastric perforating branch varices are distributed in serosal layers before and after the lesser curvature of the stomach (Photo 3). So, we emphasize an anterior-posterior pathway on completing the devascularization procedure, which is more in line with human anatomical features. Thirdly, CT imaging plays an important role in both the preoperative decision making as mentioned above and postoperative evaluation. Postoperative CT image reconstruction provides reliable proof for the integrity of the left gastric vein stem and paraesophageal vein and disappearance of periesophageal gastric perforating varices (Photo 4), which confirms the success of LSEGDS. However, not all the paraesophageal veins are visible by preoperative CT imaging. Fourthly, patients who have been treated with endoscopic sclerotherapy have an increased risk of surgical failure, because the chanced anatomical structure of the esophagus and gastric fundus make it difficult to isolate the paraesophageal vein.

In the present study, the LSEGDS group showed no difference from the LEGDS group for postoperative mortality, postoperative complications, total rebleeding rate, PVT, or encephalopathy. The LSEGDS had less blood loss. We believe that it was attributed to these improvements in surgical techniques. Although postoperative platelet counts were significantly increased in the LEGDS group, there was no increase in PVT. That is to say, postoperative platelet counts may not be a risk factor for postoperative PVT. Furthermore, the total PVT rate was $20.8 \%$ (15/72) without any anticoagulant therapy, which was lower than that previously reported [16, 17]. The postoperative esophagogastric varices rebleeding rate was low in the LSEGDS group $(6.2 \%, 2 / 32)$

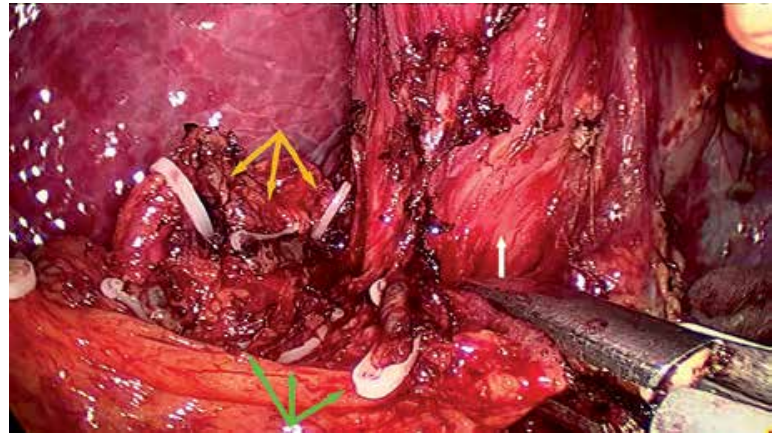

Photo 3. Distribution of gastric perforating branch varices at lesser curvature of stomach. Yellow arrowhead points to anterior serosa layer and green arrowhead points to posterior serosa layer

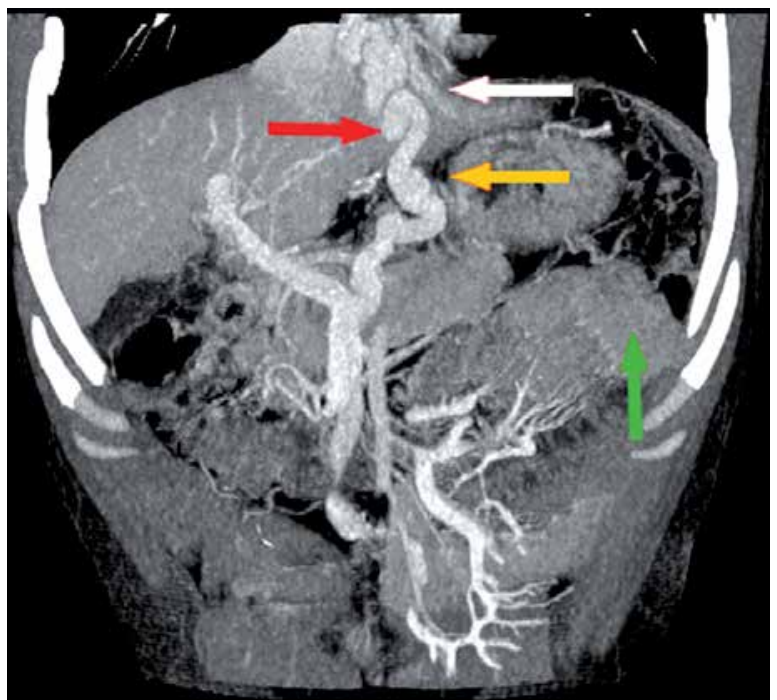

Photo 4. Postoperative CT image reconstruction of the portal vein system. White arrowhead points to esophagus; red arrowhead points to paraesophageal vein; yellow arrowhead points to disappearance of perforating branch; and green arrowhead points to removal of spleen

compared with the LEGDS group $(10 \%, 4 / 40)$ but the difference was not significant.

It should be noted that the total operative mortality was $2.7 \%(2 / 74)$ in this study. This severe complication was due to postoperative abdominal bleeding, which is life-threatening. Postoperative abdominal bleeding caused severe damage to liver function, which resulted in poor coagulation. We suggest that laparoscopic devascularization is still a high-risk procedure that should be performed by experienced laparoscopic surgeons. To avoid oper- 
ative mortality, supportive measures including improving coagulation, blood loss, liver function, nutrition, and so on, should be strengthened during the perioperative period, and the skills of the surgeon performing the laparoscopic procedure should be improved.

\section{Conclusions}

The LSEGDS is a feasible, safe and effective procedure for the management of portal hypertension resulting from liver cirrhosis, which retains a spontaneous shunt of the human body. We believe that it is especially an ideal option in patients with visible paraesophageal veins in preoperative CT scanning.

\section{Conflict of interest}

The authors declare no conflict of interest.

\section{References}

1. Luigiano C, labichino G, Judica A, Virgilio C, Peta V, Abenavoli L. Role of endoscopy in management of gastrointestinal complications of portal hypertension. World I Gastrointest Endosc 2015; 7: 1-12

2. Bari K, Garcia-Tsao G. Treatment of portal hypertension. World J Gastroenterol 2012; 18: 1166-75.

3. Chen XD, He FQ, Yang L, Yu YY, Zhou ZG. Laparoscopic splenectomy with or without devascularization of the stomach for liver cirrhosis and portal hypertension: a systematic review. ANZ J Surg 2013; 83: 122-8.

4. Wang C, Xiao L, Han J, Jin CE, Peng Y, Yang Z. A prospective randomized trial of selective versus nonselective esophagogastric devascularization for portal hypertension. J Huazhong Univ Sci Technolog Med Sci 2014; 34: 563-8.

5. Hashizume M, Tanoue K, Morita M, Ohta M, Tomikawa M, Sugimachi K. Laparoscopic gastric devascularization and splenectomy for sclerotherapy-resistant esophagogastric varices with hypersplenism. J Am Coll Surg 1998; 187: 263-70.

6. Yamamoto J, Nagai M, Smith B, et al. Hand-assisted laparoscopic splenectomy and devascularization of the upper stomach in the management of gastric varices. World I Surg 2006; 30: 1520-5.

7. Xin Z, Qingguang L, Yingmin Y. Total laparoscopic versus open splenectomy and esophagogastric devascularization in the management of portal hypertension: a comparative study. Dig Surg 2009; 26: 499-505.

8. Zhe C, Jian-Wei L, Jian C, et al. Laparoscopic versus open splenectomy and esophagogastric devascularization for bleeding varices or severe hypersplenism: a comparative study. J Gastrointest Surg 2013; 17: 654-9.

9. Hong D, Cheng J, Wang Z, et al. Comparison of two laparoscopic splenectomy plus pericardial devascularization techniques for management of portal hypertension and hypersplenism. Surg Endosc 2015; 29: 3819-26.
10. Zychowicz A, Radkowiak D, Lasek A, et al. Laparoscopic splenectomy for immune thrombocytopenia in patients with a very low platelet count. Videosurgery Miniinv 2018; 13: 157-63.

11. Zhu JH, Wang YD, Ye ZY, et al. Laparoscopic versus open splenectomy for hypersplenism secondary to liver cirrhosis. Surg Laparosc Endosc Percutan Tech 2009; 19: 258-62.

12. Cai YQ, Zhou J, Chen XD, Wang YC, Wu Z, Peng B. Laparoscopic splenectomy is an effective and safe intervention for hypersplenism secondary to liver cirrhosis. Surg Endosc 2011; 25: 3791-7.

13. Zheng X, Dou C, Yao Y, Liu Q. A meta-analysis study of laparoscopic versus open splenectomy with or without esophagogastric devascularization in the management of liver cirrhosis and portal hypertension. J Laparoendosc Adv Surg Tech A 2015; 25: 103-11.

14. Shi B, Yang Z, Wang X, et al. Selective periesophagogastric devascularization in portal hypertension: results of $56 \mathrm{pa}$ tients. Hepatogastroenterology 2009; 56: 492-7.

15. Yang, Z. Applied anatomy of selective periesophagogastric devascularization. Chin J Pract Surg 2005; 25: 702-4.

16. Cheng Z, Li JW, Chen J, Fan YD, Guo P, Zheng SG. Therapeutic effects of laparoscopic splenectomy and esophagogastric devascularization on liver cirrhosis and portal hypertension in 204 cases. J Laparoendosc Adv Surg Tech A 2014; 24: 612-6.

17. Cheng Z, Yu F, Tian J, et al. A comparative study of two anti-coagulation plans on the prevention of PVST after laparoscopic splenectomy and esophagogastric devascularization. J Thromb Thrombolysis 2015; 40: 294-301.

Received: 9.09.2018, accepted: 9.10.2018 